\title{
Vaccination in the primary care setting: when is it safe to proceed?
}

Hui Lee Sharon $\underline{\mathrm{Ngoh}}^{1}$, MBBS, MMed, Mark Chung Wai $\underline{\mathrm{Ng}}^{1}$, MMed, FCFP

The Soh family, your long-time patients, visited your clinic with their 12-month-old daughter Arial and Mr Soh's mother, Mdm Tan. Arial needed her first measles, mumps and rubella (MMR) vaccine, but Mrs Soh expressed concern about the vaccine's safety, as they had a family history of febrile seizures. Mdm Tan, a 70-year-old woman, wanted to know if she was eligible for the zoster vaccine. She was diagnosed with breast cancer a year ago, underwent surgery and is currently on tamoxifen as adjuvant therapy.

\section{HOW RELEVANT IS THIS TO MY PRACTICE?}

Vaccinations are an effective public health intervention against outbreaks of contagious diseases, which can be serious and fatal. Primary care practitioners, who play an important role in advocating and administrating both childhood and adult vaccination, are often faced with situations where the risks of administering a vaccine may seem to outweigh its benefits. In these situations, a good understanding of precautions and contraindications to vaccination helps in decision making. An earlier article on childhood immunisation highlighted some basic principles of vaccine scheduling, such as minimum age and minimum interval requirements. ${ }^{(1)}$ This article focuses on the precautions, contraindications and false contraindications to vaccine administration in both children and adults.

\section{PRECAUTIONS, CONTRAINDICATIONS AND FALSE CONTRAINDICATIONS}

The consensus among most experts is that there are very few conditions where vaccines are contraindicated. These include known hypersensitivity to one or more vaccine components, hypersensitivity to a prior dose of vaccine, encephalopathy occurring within one week of pertussis vaccination with no other identifiable cause, intussusception following rotavirus vaccination and severe combined immunodeficiency. ${ }^{(2)}$

Precautions are medical conditions that may interfere with vaccination. Although the vaccine may be given, the risks associated with vaccine administration need to be weighed against the benefits. Examples of precautions include worsening of thrombocytopenia after a dose of measles, mumps and rubella (MMR) vaccine in recipients with pre-existing idiopathic thrombocytopenic purpura, and the risk of abscess formation and lymphadenitis after the Bacillus Calmette-Guérin (BCG) vaccine. ${ }^{(3,4)}$ In these situations, the patient should be counselled appropriately and a joint decision arrived at.
Finally, there are false contraindications where the risks of administering the vaccine seem to outweigh the benefits, but in fact do not. As such, golden opportunities for vaccination can be missed or unnecessarily delayed. The following sections highlight common clinical situations where misperceptions can arise, in hopes of encouraging practitioners to make a more positive decision regarding immunisation.

False contraindication 1: children with a personal history of febrile seizures or family history of adverse events

Febrile seizures are common in childhood and can sometimes be induced by fever that develops following vaccine administration. ${ }^{(5)}$ Having a first-degree relative who has had a febrile seizure is a risk factor. ${ }^{(6)}$ However, a family history of seizures, sudden infant death syndrome or adverse reactions unrelated to immunosuppression following a dose of vaccine is not a contraindication to immunisation. ${ }^{(2)}$

Certain vaccines are associated with a small increase in the risk of febrile seizures, particularly when co-administered with some others. For instance, the risk of febrile seizures is increased when the inactivated influenza vaccine is administered with the pneumococcal 13-valent conjugate vaccine (PCV13) or diphtheria, tetanus and acellular pertussis (DTaP) vaccine. ${ }^{(5)}$ However, when given on a different day from the other two vaccines, the influenza vaccine was not associated with an increased risk of febrile seizures. ${ }^{(5)}$ Experts have recommended that patients with a personal or family history of febrile fits should be warned about the risk of febrile seizures following the MMRvaricella (MMRV) vaccine. Higher rates of fever and febrile fit events have been reported with the first dose of MMRV vaccine $(1: 1,250)$ given to children $\leq 4$ years of age, as compared to MMR and chickenpox vaccines $(1: 2,500)$ co-administered at different sites during the same visit. ${ }^{(3,7)}$ On the other hand, some febrile seizures may be prevented by protecting children against vaccine-preventable diseases that can cause fever, such as 
measles, chickenpox, influenza and pneumococcal infections. As such, it is important to ensure that childhood vaccines are not unnecessarily omitted or delayed.

False contraindication 2: individuals living with pregnant women or immunosuppressed patients

For live-attenuated vaccines, there is a rare possibility of the vaccine-strain pathogen causing disease in the recipient, who may then transmit it to susceptible household contacts (i.e. pregnant women or immunosuppressed patients). While transmission of measles and mumps vaccine viruses to household members or other close contacts has never been reported, chickenpox vaccine virus transmission has been described, but only in a few case reports. ${ }^{(2,8-10)}$ As such, administering a live-attenuated vaccine to a person living with susceptible household contacts may seem risky. However, the chance of diseases caused by a vaccine-strain virus is very remote. On the contrary, there is a higher chance of catching a wild strain of the virus. For example, the seroprevalence rate of varicella-zoster virus infection among children under the age of five years in Singapore has increased from $4 \%$ between 1989 and 1990 to 32.2\% between 2008 and 2010. ${ }^{(11}$ In addition, the household contact transmission rate for the varicella-zoster virus is approximately $85 \%{ }^{(8)}$ Hence, the most effective way to protect immunosuppressed patients or pregnant women against chickenpox is to vaccinate their close contacts. In such cases, the benefits of vaccinating the child likely outweigh the risks.

In other words, most live-attenuated vaccines can be given to those who are in close contact with pregnant women or immunosuppressed patients, with the exception of the smallpox vaccine. Some protection from herd immunity can also be conferred on those who are not eligible for live-attenuated vaccines.

\section{False contraindication 3: breastfeeding mothers}

No vaccines are contraindicated in breastfeeding, with the exception of the yellow fever vaccine.(2) However, breastfeeding mothers cannot avoid or postpone yellow fever vaccination if they are travelling to high-risk yellow fever-endemic areas. ${ }^{(12)}$ The rubella vaccine virus may be present in the breast milk of a vaccinated woman, but transmission of infection to the infant is rare. ${ }^{(2)}$ Even if transmission does occur, the resultant disease is well-tolerated, as the viruses are attenuated. ${ }^{(12)}$

\section{False contraindication 4: patients who may be pregnant} and require inactivated vaccines

Live-attenuated vaccines are contraindicated in pregnancy, whereas inactivated vaccines can be safely administered to pregnant woman, with the exception of the human papilloma virus (HPV) vaccine. To date, safety data on the use of the HPV vaccine in pregnant patients is lacking. ${ }^{(13)}$ For example, if a young newlywed couple who are trying for a child request for chickenpox, hepatitis $A$, injectable typhoid and influenza vaccines, all vaccines can be given to the woman except for the chickenpox vaccine. The chickenpox vaccine is a live-attenuated vaccine, while the rest are not.

Pregnant women are strongly recommended to receive the inactivated influenza vaccine because they are more likely to develop severe disease with influenza. ${ }^{(12)}$ Administration of the tetanus toxoid, reduced diphtheria toxoid and acellular pertussis (Tdap) vaccine is recommended between 27 and 36 weeks of gestation, although it can be given at any time during pregnancy. ${ }^{(2)}$ Pertussis, which is highly transmissible through close contact and respiratory droplets, is one of the most common vaccinepreventable diseases. ${ }^{(14)}$ It can cause very serious illness in babies. ${ }^{(14)}$ Tdap should be administered during pregnancy so that passive immunity can be conferred on the child. ${ }^{(15)}$ If Tdap is not administered during the pregnancy for any reason, it should be given postpartum. ${ }^{(15)}$

\section{False contraindication 5: patients recently on a course of antibiotics}

Antibiotics generally do not affect the immune response to most vaccines, although there are some exceptions. The Ty21a oral typhoid vaccine should be delayed for at least 72 hours in patients who have received sulphonamides or other antibiotics. Similarly, patients receiving antiviral treatment should only receive the influenza vaccine 48 hours after the cessation of therapy. ${ }^{(2)}$ After discontinuing antiviral therapy for treatment of the herpes virus, a 24-hour wait is recommended before administering the chickenpox vaccine. ${ }^{(2}$

\section{False contraindication 6: patients on corticosteroids}

Inactivated vaccines are not contraindicated in patients who are on aerosolised steroids, short tapering courses of steroids, topical steroids or steroid doses for physiological replacement. Some primary care practitioners might be concerned about the safety of administering a live-attenuated vaccine shortly after the patient has received high-dose steroids. In fact, live-attenuated vaccines should only be deferred if the patient has received high-dose steroids ( $\geq 20 \mathrm{mg}$ prednisolone daily or $\geq 2 \mathrm{mg} / \mathrm{kg} /$ day) for 14 or more days. ${ }^{(2)}$ For example, a patient who has just completed a seven-day course of high-dose prednisolone for acute asthma exacerbation can be given the chickenpox or zoster vaccine.

False contraindication 7: patients who had chemotherapy previously or who are currently on hormonal therapy for cancer treatment

Some primary care practitioners may be concerned about administering live-attenuated vaccines to patients who previously had chemotherapy. In general, live-attenuated vaccines can be given three months after chemotherapy is completed or discontinued.(2) However, as practices may differ across institutions, it is prudent for the general practitioner to discuss treatment options with the patient as well as their specialists before making the decision to vaccinate. Patients on adjuvant cancer treatment such as tamoxifen or gonadotropin-releasing hormone inhibitors can receive live-attenuated or inactivated vaccines. 
These hormonal therapies do not affect the immune system and do not cause severe immunosuppression. ${ }^{(16)}$

\section{False contraindication 8: patients with human immunodeficiency virus (HIV) infection}

As persons with HIV infection are generally immunocompromised, there may be concerns regarding the administration of liveattenuated vaccines. In general, live-attenuated vaccines are usually contraindicated in those who are severely immunosuppressed. However, for asymptomatic HIV-positive individuals who are less severely immunocompromised (CD4 count $\geq 200$ cells $/ \mathrm{mm}^{3}$ ), the chickenpox vaccine can be considered. ${ }^{(17)}$ Similarly, the yellow fever vaccine should be considered for individuals with no symptomatic manifestation of HIV infection or acquired immunodeficiency syndrome (AIDS), who have laboratory confirmation of adequate function of the immune system and are at a high risk of being exposed to the yellow fever virus. ${ }^{(2)}$ As for inactivated vaccines, they do not pose a risk to immunosuppressed persons and should be used for the same indications seen in healthy individuals. ${ }^{(18)}$ In fact, since influenza and pneumococcal infections may result in severe sequelae in immunocompromised persons, including those with HIV infection, vaccination against these two diseases is highly recommended. ${ }^{(17,19)}$

\section{False contraindication 9: patients with recent vaccination}

A history of recent immunisation is generally not a contraindication to receiving another vaccine. Table I shows vaccines commonly given in the primary care setting. However, there are certain noteworthy exceptions. If two live-attenuated vaccines are not administered simultaneously, they need to be spaced apart by at least four weeks. ${ }^{(2)}$ This is to reduce or eliminate the first vaccine's interference with the patient's antibody response to the second live-attenuated vaccine. If the second live-attenuated vaccine is administered earlier than the recommended minimum interval, it should be repeated in 28 days from the date of the invalid dose, with the exception of the yellow fever vaccine. ${ }^{(2)}$

For inactivated vaccines, no interval is necessary to administer the second live-attenuated or inactivated vaccine. One exception to this is PCV13 and the pneumococcal polysaccharide vaccine (PPSV23), which should be given apart. The required interval between these two vaccines depends on the patient's age and underlying medical condition(s), as well as the sequence of administration. In children with risk factors (e.g. functional or anatomical asplenia, cerebrospinal fluid leak, cochlear implants or immunocompromised states) aged 24 months to 18 years, a minimum interval of eight weeks is recommended between PCV13 and PPSV23, regardless of the sequence of administration. ${ }^{(20)}$ For adults aged $\geq 65$ years, PPSV23 is given 12 months after PCV13. ${ }^{(20)}$ Adults aged $\geq 19$ years with risk factors (e.g. functional or anatomic asplenia, cerebrospinal fluid leaks, cochlear implants or immunocompromised states) should be given PPSV23 no earlier than eight weeks after PCV13. ${ }^{(20)}$ For individuals aged $\geq 19$ years, if PPSV23 is given earlier than PCV13 for any reason, it is recommended that PCV13 be delayed for least 12 months. ${ }^{(20)}$
Table I. List of live-attenuated and inactivated vaccines commonly used in primary care.

\begin{tabular}{ll}
\hline Live-attenuated & Measles, mumps and rubella vaccine \\
& Chickenpox vaccine \\
Zoster vaccine & Rotavirus vaccine \\
& Yellow fever vaccine \\
& Oral typhoid vaccine \\
& Bacillus Calmette-Guérin vaccine \\
& Hepatitis A vaccine \\
& Diphtheria, pertussis and tetanus vaccine \\
Inactivated & Tetanus toxoid \\
& Hepatitis B vaccine \\
Influenza vaccine & Pneumococcal conjugate vaccine \\
& Pneumococcal polysaccharide vaccine \\
& Meningococcal conjugate vaccine \\
& Meningococcal polysaccharide vaccine \\
Human papilloma virus vaccine
\end{tabular}

\section{CONCLUSION}

There are many situations where the primary care practitioner may feel compelled to defer or decline administering a vaccine. It is important that vaccines that are indicated should be offered and administered to patients in a timely manner, without unnecessary delay. When in doubt, the primary care doctor should seek expert opinion, discuss the merits and drawbacks of a particular vaccine with the patient and arrive at a decision made in the best interest of the patient. This will eliminate many missed opportunities and improve immunisation coverage rates, ultimately increasing herd immunity and protecting the general population as well as the vaccinated individuals.

You addressed Mrs Soh's concerns regarding the safety of the MMR vaccine, explaining that a family history of febrile seizures is neither a contraindication nor a precaution. Although there is a theoretical risk, the transmission of measles and mumps vaccine viruses has never been reported. Therefore, it is safe for Arial to receive the MMR vaccine. Mdm Tan can also receive the zoster vaccine, as tamoxifen would not cause immunosuppression and does not affect the efficacy of the vaccine.

\section{TAKE HOME MESSAGES}

1. Most live-attenuated vaccines can be given to individuals living with pregnant women or immunosuppressed persons.

2. With the exception of the yellow fever vaccine, breastfeeding is not a contraindication for any vaccination.

3. Generally, vaccination is not contraindicated in patients who are on antibiotics, aerosolised steroids, topical steroids, short, tapering courses of steroids and physiological steroid replacements.

4. Live-attenuated vaccines should not be given to patients 
who are on high doses of prednisolone over a prolonged period of time.

5. Live-attenuated vaccines are usually contraindicated in severely immunosuppressed patients, but inactivated vaccines are not.

6. Most inactivated or live-attenuated vaccines can be given simultaneously at the same visit, with some exceptions.

7. If two live-attenuated vaccines are not given simultaneously, ensure a minimum interval of 28 days before administering the second live-attenuated vaccine.

8. The reported rates of fever and febrile fit events with the first dose of MMRV vaccine given to children $\leq 4$ years of age are higher compared to MMR and chickenpox vaccines co-administered at different sites during the same visit.

ABSTRACT Primary care practitioners play an important role in administering and advocating vaccinations against vaccine-preventable infectious diseases and ensuring herd immunity in our population. This is a follow-up article to an earlier one which dealt with the principles of vaccine scheduling and administration. This article describes several false contraindications to vaccination that a primary care practitioner may encounter, including pregnancy, current breastfeeding, history of febrile seizures, and having immunosuppressed or pregnant household contacts. We aimed to provide a guide for safe and timely vaccine administration in the primary care setting.

Keywords: false contraindication, primary care, vaccination

\section{REFERENCES}

1. Ng MC, How CH. Childhood immunisation. Singapore Med J 2014; 55:12-7.

2. Centers for Disease Control and Prevention. Chapter 2: General Recommendations on Immunization. In: Hamborsky J, Kroger A, Wolfe S, eds. Epidemiology and Prevention of Vaccine-Preventable Diseases. 13th ed. Washington DC: Public Health Foundation, 2015 [online]. Available at: http://www.cdc.gov/vaccines/pubs/pinkbook/genrec.html. Accessed November 10, 2015.

3. Thoon KC, Chong CY, Lim KS, Seet CM, Stephanie J. KKH Vaccine Pocketbook. Singapore: KK Women's and Children's Hospital, 2015.

4. Cecinati V, Principi N, Brescia L, Giordano P, Esposito S. Vaccine administration and the development of immune thrombocytopenic purpura in children. Hum Vaccin Immunother 2013; 9:1158-62.5.

5. Centers for Disease Control and Prevention. Childhood vaccines and febrile seizures [online]. Available at: http://www.cdc.gov/vaccinesafety/ concerns/febrile-seizures.html. Accessed December 1, 2015.

6. Graves RC, Oehler K, Tingle LE. Febrile seizures: risks, evaluation, and prognosis. Am Fam Physician 2012; 85:149-53.
7. Centers for Disease Control and Prevention. Use of Combination Measles, Mumps, Rubella, and Varicella Vaccine: Recommendations of the Advisory Committee on Immunization Practices. In: Morbidity and Mortality Weekly Report/Vol.59/No.RR-3. 7 May 2010; 3-5 [online]. Available at: http:// www.cdc.gov/mmwr/pdf/rr/rr5903.pdf. Accessed December 1, 2015.

8. Centers for Disease Control and Prevention. Prevention of Varicella: Recommendations of the Advisory Committee on Immunization Practices (ACIP). In: Morbidity and Mortality Weekly Report/Vol.56/No.RR-04. 22 June 2007; 1-40 [online]. Available at: http://www.cdc.gov/mmwr/preview/ mmwrhtml/rr5604a1.htm. Accessed December 5, 2015.

9. Salzman MB, Sharrar RG, Steinberg S, LaRussa P. Transmission of varicellavaccine virus from a healthy 12 -month-old child to his pregnant mother. J Pediatr 1997; 131:151-4.

10. LaRussa P, Steinberg S, Meurice F, Gershon A. Transmission of vaccine strain varicella-zoster virus from a healthy adult with vaccine-associated rash to susceptible household contacts. J Infect Dis 1997; 176:1072-5.

11. Fatha N, Ang LW, Goh KT. Changing seroprevalence of varicella zoster virus infection in a tropical city state, Singapore. Int J Infect Dis 2014; 22:73-7.

12. Centers for Disease Control and Prevention. Vaccines and Immunizations. Guidelines for Vaccinating Pregnant Women [online]. Available at: http:// www.cdc.gov/vaccines/pubs/preg-guide.htm. Accessed December 29, 2015.

13. Centers for Disease Control and Prevention. FDA Licensure of Bivalent Human Papillomavirus Vaccine (HPV2, Cervarix) for Use in Females and Updated HPV Vaccination Recommendations from the Advisory Committee on Immunization Practices (ACIP). In: Morbidity and Mortality Weekly Report/Vol.59/No.20. 28 May 2010; 626-9 [online]. Available at: http://www.cdc.gov/mmwr/pdf/wk/mm5920.pdf. Accessed December 5, 2015.

14. Centers for Disease Control and Prevention. Pertussis (Whooping Cough): Complications [online]. Available at: http://www.cdc.gov/pertussis/about/ complications.html. Accessed December 29, 2015.

15. Centers for Disease Control and Prevention. Updated Recommendations for Use of Tetanus Toxoid, Reduced Diphtheria Toxoid, and Acellular Pertussis Vaccine (Tdap) in Pregnant Women — Advisory Committee on Immunization Practices (ACIP), 2012. In: Morbidity and Mortality Weekly Report/Vol.62/No.7. 22 February 2013; 131-13 [online]. Available at: http://www.cdc.gov/mmwr/pdf/wk/mm6207.pdf. Accessed December 6, 2015.

16. Public Health Agency of Canada. Canadian Immunization Guide. Part 3: Vaccination of Specific Populations. Immunization of Immunocompromised Persons [online]. Available at: http://www.phac-aspc.gc.ca/publicat/ciggci/p03-07-eng.php. Accessed December 3, 2015.

17. Centers for Disease Control and Prevention. Vaccine Information for Adults: HIV Infection and Adult Vaccination [online]. Available at: http://www. cdc.gov/vaccines/adults/rec-vac/health-conditions/hiv.html. Accessed December 29, 2015

18. Centers for Disease Control and Prevention. Recommendations of the Advisory Committee on Immunization Practices (ACIP): Use of Vaccines and Immune Globulins in Persons with Altered Immunocompetence. In: Morbidity and Mortality Weekly Report/Vol.42/No.RR-4. 9 April 1993; 1-18 [online]. Available at: http://www.cdc.gov/mmwr/pdf/rr/rr4204.pdf. Accessed December 29, 2015.

19. Zbinden D, Manuel O. Influenza vaccination in immunocompromised patients: efficacy and safety. Immunotherapy 2014; 6:131-9.

20. Centers for Disease Control and Prevention. Intervals Between PCV13 and PPSV23 Vaccines: Recommendations of the Advisory Committee on Immunization Practices (ACIP). In: Morbidity and Mortality Weekly Report/Vol.64/No.34. 4 September 2015; 944-7 [online]. Available at: http://www.cdc.gov/mmwr/pdf/wk/mm6434.pdf. Accessed December 3, 2015. 


\section{SINGAPORE MEDICAL COUNCIL CATEGORY 3B CME PROGRAMME} (Code SMJ 201601A)

1. Intussusception following rotavirus vaccination is a contraindication to the subsequent dose.

2. The subsequent dose of measles, mumps, and rubella (MMR) vaccine is contraindicated in patients with idiopathic thrombocytopenic purpura whose thrombocytopenia worsened after the first dose.

3. In general, there are very few contraindications in which the risks of administering the vaccine outweigh the benefits.

4. A strong family history of febrile seizures is a contraindication to vaccination, especially when previous vaccination resulted in a post-vaccination fever.

5. A previous episode of febrile seizures is a contraindication to the MMR-varicella (MMRV) vaccine.

6. There are greater rates of fever and febrile fits following administration of the MMRV vaccine as compared to MMR and chickenpox vaccines co-administered at separate sites during the same visit.

7. A family history of sudden infant death syndrome is an absolute contraindication to immunisation.

8. Transmission of the vaccine-strain virus to susceptible household contacts is commonly seen after receiving live-attenuated vaccines, such as the chickenpox or MMR vaccines.

9. Most live-attenuated vaccines can be given to individuals who are living with pregnant women or mildly immunosuppressed persons.

10. After administration of a live-attenuated vaccine, breastfeeding is contraindicated for the next 48 hours to prevent the baby from catching vaccine-associated infections.

11. It is generally safe to administer inactivated vaccines to pregnant women, with the exception of the human papilloma virus vaccine.

12. When administered during pregnancy, the tetanus toxoid, reduced diphtheria toxoid and acellular pertussis (Tdap) vaccine can confer passive immunity on the child.

13. The Ty21a oral typhoid vaccine should not be given to patients on antibiotic therapy until 72 hours have lapsed.

14. Patients receiving antiviral treatment should only receive the influenza vaccine 48 hours after the cessation of therapy.

15. Patients who are on a short, tapering course of steroids will have a reduced immune response to any vaccination they receive.

16. Live-attenuated vaccines should be deferred if the patient has received high-dose steroids ( $\geq 20 \mathrm{mg}$ prednisolone daily or $\geq 2 \mathrm{mg} / \mathrm{kg} /$ day) for 14 days or more.

17. An asthmatic who has just completed a seven-day course of high-dose prednisolone for acute exacerbation can receive the chickenpox vaccine at any time if there are no other contraindications.

18. Patients on adjuvant cancer treatment such as tamoxifen must defer vaccination for a month from their last dose.

19. In cases where both are indicated, the interval between the pneumococcal conjugate vaccine (PCV13) and pneumococcal polysaccharide vaccine (PPSV23) depends on the patients' age and underlying medical condition, and the sequence of administration.

20. For patients aged 19 years and above, if PPSV23 is given first for any reason, it is recommended to delay PCV13 by least 12 months.

\section{Doctor's particulars:}

Name in full

MCR number

Email address

Specialty:

\section{SUBMISSION INSTRUCTIONS:}

(1) Log on at the SMJ website: http://www.sma.org.sg/publications/smjcurrentissue.aspx and select the appropriate set of questions. (2) Provide your name, email address and MCR number. (3) Select your answers and click "Submit".

RESULTS:

(1) Answers will be published in the SMJ March 2016 issue. (2) The MCR numbers of successful candidates will be posted online at the SMJ website by 4 March 2016. (3) Passing mark is $60 \%$. No mark will be deducted for incorrect answers. (4) The SMJ editorial office will submit the list of successful candidates to the Singapore Medical Council. (5) One CME point is awarded for successful candidates.

Deadline for submission: (January 2016 SMJ 3B CME programme): 12 noon, 26 February 2016. 